\title{
Aberration-corrected Scanning Transmission Electron Microscopy and Spectroscopy of Nonprecious Metal Nanoparticles in Titania Aerogels
}

\author{
Todd H. Brintlinger, ${ }^{1}$ Paul A. DeSario, ${ }^{2}$ Jeremy J. Pietron, ${ }^{2}$ Rhonda M. Stroud, ${ }^{1}$ and Debra R. Rolison ${ }^{2}$ \\ 1. Materials Science and Technology Division, U.S. Naval Research Laboratory, Washington, DC, USA \\ 2. Chemistry Division, U.S. Naval Research Laboratory, Washington, DC, USA
}

By exhibiting high surface area and efficient photochemistry, titania aerogels are being considered as potential photocatalysts for solar energy conversion ${ }^{1}$ and environmental remediation ${ }^{2}$. However, titania's large bandgap does not efficiently match the main solar spectrum; hence, development of titania-based systems active in the visible has been a significant thrust in recent materials research. One approach is to add metal nanoparticles with local surface plasmon resonances to titania aerogel so that incident photons are absorbed at lower energies. The effects of the as-incorporated metal nanoparticle size, shape, and distribution on the photocatalytic activity have been well documented for a variety of semiconductors. ${ }^{1}$ The behavior of the supported metal nanoparticles following synthesis is critical, because even small, initially well-dispersed metal nanoparticles can oxidize, aggregate, or disperse over time or upon extended illumination and exposure to ambient. With such concerns, transmission electron microscopy is ideally suited to characterize these emerging photocatalytic metal-nanoparticle/titania-aerogel composites: the metal nanoparticles are typically in the few-nanometer range and often have a metal/oxide junction that requires additional investigation to properly understand the photochemistry of these complex systems.

Following our work on composite precious metal-loaded aerogels ${ }^{3}$ we extend our investigations of these systems using nonprecious metals incorporated within titania aerogels. Following similar synthesis and photocatalytic characterization from our previous work, we find well-dispersed nonprecious metal nanoparticles within a titania matrix. The comparatively lower atomic number of nonprecious metals $(Z<30)$ and smaller overall nanoparticle size $(<4 \mathrm{~nm})$ made conventional TEM characterization a challenge, although the lattice fringes obtained from HRTEM (a JEOL JEM2200FS operating at $200 \mathrm{keV}$ ) did provide the initial indication for the presence of unoxidized metal nanoparticles within the matrix (Fig. 1). Here, an inverse fast-fourier-transform (FFT) of an FFT mask near known metallic lattice constants gives the location of a suspected metallic nanoparticle. We further corroborated these data with an aberration-corrected scanning transmission electron microscope (STEM, a Nion UltraSTEM-200X also operating at $200 \mathrm{kV}$, with a windowless Bruker SDD with 0.7 sr and Gatan Enfinium ER) using high-angle annular dark-field imaging, together with site-specific energy dispersive spectroscopy (EDS) (Fig. 2), and electron energy-loss spectroscopy (EELS, not shown here for intellectual property reasons, but which will be presented at meeting). Thus, we characterize these nonprecious metal nanoparticles and show how titania aerogels can stabilize nonprecious metal nanoparticles against oxidation, mobilization, and aggregation.

\section{References:}

[1] S. Linic, P. Christopher and D. B. Ingram, Nat. Mater. 10, p. 911 (2011).

[2] T. L. Thompson and J. T. Yates, Jr., Chem. Rev. 106, p. 4428 (2006).

[3] J. J. Pietron, R. M. Stroud and D.R. Rolison, Nano Lett. 2, p. 545 (2002). 


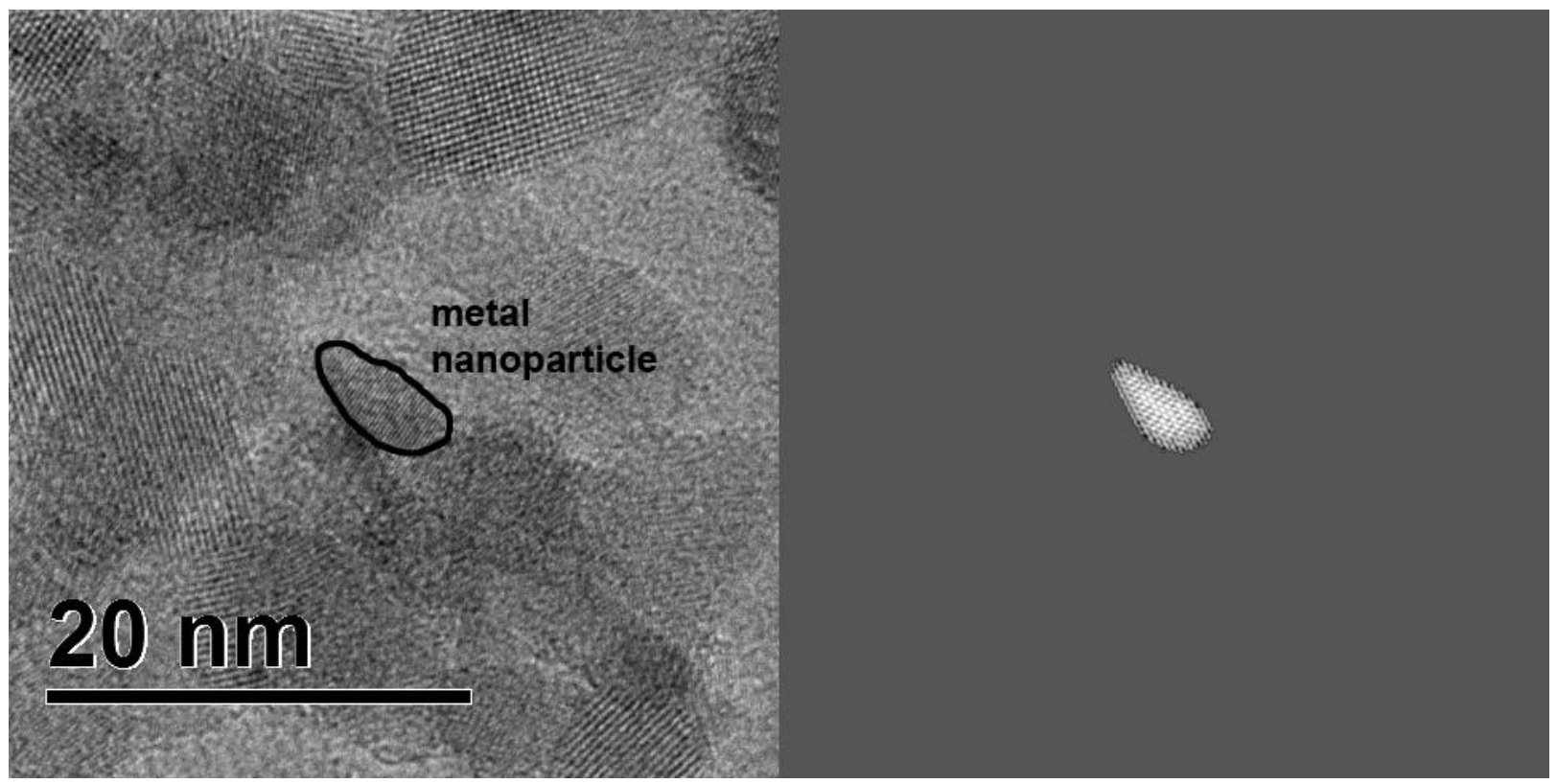

Figure 1. High-resolution transmission electron microscopy (HRTEM) of a metal nanoparticle supported within an ultraporous titania aerogel. The metallic nanoparticle (outlined in HRTEM, left) was identified using a fast-fourier transform (FFT) technique in which FFT is masked near known metallic lattice constants and then an inverse FFT is performed (right).



Figure 2. High-angle annular dark-field image (left) and energy dispersive spectroscopy (EDS) composite RGB elemental map (right) of the titania aerogel-metal nanoparticle composite. On right, each pixel is color coordinated for the spectroscopic signature, with red, green, and blue representing oxygen, titanium, and our given metal, respectively. The circled region shows a metal particle, indicating robustness against oxidation. 